\title{
Optimizing on Mobile Usage Cost for the Lower Income Group: Insights and Recommendations
}

\author{
Deepak P. and Anuradha Bhamidipaty \\ IBM India Research Lab, Bangalore, India \\ \{deepak.s.p, abhamidi\}@in.ibm.com
}

\begin{abstract}
There is an increasing trend in the penetration of mobile phones towards the lower strata (lower income) group of the society. Cost is perceived as the governing factor which determines the adoption of mobile phones in this group. This paper explores the effect of cost on the usage of mobile phones and proposes an enhanced design with features that optimize its usage cost for lower income group. These features help determine and restrict call duration, proactively alert user on usage deviations and avoid early call terminations. Preliminary evaluations of the enhanced design were decidedly positive about the effectiveness in controlling and optimizing mobile usage cost.
\end{abstract}

\section{Introduction}

Developing countries like India and China are increasingly noticing the unbalanced spread and reach of information industry infrastructure, which aggravates the urbanrural, rich-poor divide. Penetration of Information and Communication Technologies (ICT), mobile telephony in particular is viewed as an opportunity to bridge this gap [1]. We recognize that there are two aspects to taking Information Technology (IT) to the lower strata of the society; the availability and support of physical infrastructure and other barriers that prevents the penetration of technology. These barriers include illiteracy, socio-cultural effects, language barriers and cost. Multiple studies on penetration barriers have concluded that cost (or pre-conceived notions about it) is the largest impediment to adoption of mobile phones in developing countries ([2], [3]).

In this paper, we study the effect of cost on the penetration and usage of mobile phones and identify the significant cost-related pain points in mobile usage in the lower strata of the society. Using a mobile phone involves the one time cost of owing a mobile handset and the cost of maintaining it. The second factor is of more concern to the lower income group because of its recurring nature. We propose features addressing the identified pain points to be included in the "cheap-usage" mobile phone, and validate the importance of cost as a barrier to penetration of technology by gauging the acceptance of the proposed features among the lower-income group. We expect that the design of the proposed features provide insights into how technology has to be sensitive to the cost factor, if it hopes that the lower strata of the society would be able to leverage it. 


\section{User Study}

A user study of the lower income group was conducted to get insight into their spending pattern for mobile phone. Our aim was to understand the cost factor and its effect on mobile usage such as their call patterns, and as to how they managed their mobile usage budgets. The survey population consisted of 30 semi-literate mobile phone users from the lower income category in and around Bangalore, a city in the state of Karnataka in India. By semi-literate, we mean that they are able to recognize English numerals, but are not very comfortable about reading and writing the language their mobile phone uses (which was English in all the 30 cases). They had an average daily income of 5-6\$, were in their 20 s or early 30 s and were mostly from high-school dropouts who had to start earning in their teens due to poverty. Most of these people use the least expensive of the mobile phones available in the market.

\subsection{Results and Inferences}

It was seen that the usage patterns of most of the surveyed people were very similar and were very different from the usage patterns of the higher income groups. The results showed that people in lower income group spend as low as 3-15\$ per month on mobile usage with an average of $5 \$$. We enumerate our observations of the effect of cost on mobile usage as follows:

1. Preference for Pre-paid Mobile Connections: The strong conviction to keep mobile usage cost in check leads to almost all people to go in for pre-paid connections. Pre-paid connections require advance payment, and thus are effective to constrain unrestricted mobile usage.

2. Short Call Durations: Mobile phones are mostly used for conveying to-the-point information and hence tend to be very short. Long calls are usually scheduled and planned at regular intervals (e.g., weekly).

3. Skewed Call Distribution: Most of the participants reported a high skew in call distribution, with the number of contacts used on a daily basis being as low as 2-8 with an average of 4 (roughly around $5 \%$ of the size of the average contact list). Regularly contacted contacts were mostly family members and those from the inner circle of friends. This is mostly because of mobiles being perceived as devices to convey critical information only (due to the cost involved)

4. Usage of Missed Calls to Convey Information: With the widespread deployment of CLIP (Caller Line Identification), missed calls (which are not charged) have been used to convey pre-agreed Boolean information such as "I will give a missed call before I start home from workplace"

5. Urge to optimize on Call Charges by controlling call durations: Call charge is determined in terms of the number of call pulses it constitutes; each pulse has fixed duration and cost. Most participants reported that they try to check cost by trying to estimate the number of call pulses and hence the overall call duration when using the mobile phone and controlling it.

From the observations and inputs about the effects of cost on mobile usage, we identified the following as the cost related pain points of mobile usage on the lower income group 
- Determining when to end the call to optimize on call cost

- Managing mobile usage budget, especially at a finer granularity than monthly (monthly being the usual frequency of mobile related payments)

- Avoiding early call termination and voice breaks in call due to erratic signal strengths and low battery charge (both these scenarios lead to repetitive and/or unfruitful calls)

In the following section, we design features for the mobile phone to address the above mentioned pain points.

\section{Design of Mobile Phone for Cost-Effective Usage}

Based on the findings of the user study we propose an enhanced design of the mobile phone with features that optimize the mobile usage cost for the lower income group. These features listed below focus on creating "cheap-usage" mobile phone that help the user reduce call duration, avoid early call termination in some cases, and provide the intelligence to keep a proactive check on cost. The features can be implemented with current technology in mobile phones.

1. Pre-Pulse: This feature is designed to notify the user with a beep before the current call pulse changes. For example, the phone can be configured to beep 5 seconds before the pulse switch so that the user can hang up before the pulse actually switches. This lets the user talk cost effectively. Currently some mobile phone models indicate a beep sound at the pulse change, hanging up at which time leads to wasted call charge for the additional call pulse (i.e., the beep indicates when not to hang up, if cost optimization is a concern). The user can configure the phone with his pulse duration and pre-pulse duration (i.e., the number of seconds before the pulse change that the phone should beep) as a one time activity. This feature aids cost optimized usage of mobile phones. This optimizes only the last pulse of a call, and hence the effect of this optimization would be much higher on the lower strata as they usually make very short calls.

2. Preset Call Duration: This feature in the mobile phone facilitates the user to preset the duration of a call before making the call, and the call gets automatically disconnected after the said duration. For example, a user can pre-determine that conveying a particular message (short one) should not take more than a minute and can pre-set it. Aligning the pre-set call duration with the call pulse duration provides the maximum talk time for a given cost. Pre-setting call duration helps control call durations and in turn optimizes the call charges.

3. Proactive Alerts on Cost Usage: Almost all of the survey population reported a fixed allocation of budget every month for mobile usage with very little scope of accommodating any extra usage cost. An insight into their mobile call logs showed us that their call usage pattern follows a daily pattern with some variations during holidays. The idea on providing proactive alerts is to embed intelligence into the mobile phone to be able to detect deviation from the usual call usage pattern on a daily basis, which might lead to higher cost and alert the user proactively to take check on cost usage. This prevents the user from incurring usage cost which is not aligned with their spending capacity or allocated budget. 
4. Avoid Early Call Termination: This features helps avoid early call termination attributed to weak signal strength or low battery charge. If a user is attempting to make a phone call and the signal strength is weak, the mobile phone gives an alert to the user that the call may result in early termination or voice breaks, and optionally places the call details in a queue and auto connects (dials in itself) the call when the signal strength improves. The phone, optionally, alerts the user when an auto-connect is due to happen. This is critical because of erratic signal strengths due to poor mobile service infrastructure in the rural areas. Similarly, if the mobile has low battery charge and the user is about to make a long phone call (as he indicates using a pre-set call duration), an alert is signaled to the user to defer the call as it could possibly result in an early call termination.

\section{Evaluation, Conclusions and Future Work}

We conducted preliminary evaluation of our enhanced mobile design with 10 participants to obtain qualitative feedback on usefulness of added features and their effectiveness in controlling the mobile cost usage. The participants were drawn from the same population that was used for our user study. We explained the additional features to each of the participants and asked them to comment on what the enhancement meant to them. Interestingly, all the participants were decidedly positive about any feature in general that could help control mobile usage cost.

Of these four features, the survey population was most excited about the Pre-Pulse and Preset Call Duration features. These led to cost optimizations that they were already trying to practice manually, and hence, the automated solutions were immediately appealing to them. Incidentally, they reported an overrun of planned call duration around $20 \%$ of the time. Almost half of the survey population were unable to grasp the feature on proactive alerts well, and had concerns about whether an automated alert system was possible at all. They were receptive to the idea of tracking day-to-day mobile usage, but felt that alerts on remaining balance on a daily basis was itself very informative. All the participants found the idea on avoiding early call termination very exciting, especially because they live in areas of very erratic signal strengths. The proposed design can be achieved with current technology and would help the people in the lower strata of the society realize direct improvement over what is being offered today. We are currently working on optimizing cost on other usages of the mobile phone such as messaging and ring tones.

\section{References}

1. Sinha, C.: Effect of Mobile Telephony on Empowering Rural Communities in Developing Countries. In: Conference on Digital Divide and Global Development (November 2006)

2. Jhunjhunwala, A.: Can Telecom and IT be for the Disadvantaged?, http://www.tenet.res.in/ Papers/Tel-IT/TelecomAndIT.html

3. Blixt, P.: Mobile Telephony in Rural India. Adapting the mobile telephone to the conditions of the unprivileged rural India, Master's Thesis, Royal Inst. of Tech., Sweden (2005) 